\title{
Detection of gray matter microstructural changes in Alzheimer's disease continuum using fiber orientation
}

Peter Lee ${ }^{1,2}$, Hang-Rai Kim ${ }^{2,3}$, Yong Jeong ${ }^{1,2,3^{*}}$ (D) and for the Alzheimer's Disease Neuroimaging Initiative

\begin{abstract}
Background: This study aimed to investigate feasible gray matter microstructural biomarkers with high sensitivity for early Alzheimer's disease (AD) detection. We propose a diffusion tensor imaging (DTI) measure, "radiality", as an early AD biomarker. It is the dot product of the normal vector of the cortical surface and primary diffusion direction, which reflects the fiber orientation within the cortical column.
\end{abstract}

Methods: We analyzed neuroimages from the Alzheimer's Disease Neuroimaging Initiative (ADNI) database, including images from 78 cognitively normal (CN), 50 early mild cognitive impairment (EMCl), 34 late mild cognitive impairment (LMCI), and 39 AD patients. We then evaluated the cortical thickness (CTh), mean diffusivity (MD), which are conventional AD magnetic resonance imaging (MRI) biomarkers, and the amount of accumulated amyloid and tau using positron emission tomography (PET). Radiality was projected on the gray matter surface to compare and validate the changes with different stages alongside other neuroimage biomarkers.

Results: The results revealed decreased radiality primarily in the entorhinal, insula, frontal, and temporal cortex with further progression of disease. In particular, radiality could delineate the difference between the CN and EMCl groups, while the other biomarkers could not. We examined the relationship between radiality and other biomarkers to validate its pathological evidence in AD. Overall, radiality showed a high association with conventional biomarkers. Additional ROI analysis revealed the dynamics of AD-related changes as stages onward.

Conclusion: Radiality in cortical gray matter showed AD-specific changes and relevance with other conventional AD biomarkers with high sensitivity. Moreover, radiality could identify the group differences seen in EMCl, representative of changes in early $\mathrm{AD}$, which supports its superiority in early diagnosis compared to that possible with conventional biomarkers. We provide evidence of structural changes with cognitive impairment and suggest radiality as a sensitive biomarker for identifying early AD.

Keywords: Alzheimer's disease, Early diagnosis, Diffusion tensor imaging, Microstructure, Biomarkers

\footnotetext{
* Correspondence: yong@kaist.ac.kr

${ }^{1}$ Department of Bio and Brain Engineering, Korea Advanced Institute of

Science and Technology, Daehak-ro 291, Yuseong-gu, Daejeon 34141,

Republic of Korea

${ }^{2}$ KI for Health Science and Technology, Korea Advanced Institute of Science

and Technology, Daejeon, Republic of Korea

Full list of author information is available at the end of the article
}

\section{$\triangle B M C$}

(c) The Author(s). 2020 Open Access This article is licensed under a Creative Commons Attribution 4.0 International License, which permits use, sharing, adaptation, distribution and reproduction in any medium or format, as long as you give appropriate credit to the original author(s) and the source, provide a link to the Creative Commons licence, and indicate if changes were made. The images or other third party material in this article are included in the article's Creative Commons licence, unless indicated otherwise in a credit line to the material. If material is not included in the article's Creative Commons licence and your intended use is not permitted by statutory regulation or exceeds the permitted use, you will need to obtain permission directly from the copyright holder. To view a copy of this licence, visit http://creativecommons.org/licenses/by/4.0/ The Creative Commons Public Domain Dedication waiver (http://creativecommons.org/publicdomain/zero/1.0/) applies to the data made available in this article, unless otherwise stated in a credit line to the data. 


\section{Background}

Alzheimer's disease (AD) is notorious for its long preclinical period where various pathophysiological changes occur before the main symptoms develop. As the progression of $\mathrm{AD}$ is not completely understood, early diagnosis and intervention remain challenging [1, 2]. Repetitive failures of recent drug trials are attributed to the application of treatment to patients with relatively advanced disease [3-5]. Thus, identification of people in the earlier stages of pathology is critical in clinical trials and may be promising for controlling this devastating disease.

At present, several biomarkers are used to diagnose and monitor disease progression: amyloid and tau deposits based on positron emission tomography (PET) imaging or cerebrospinal fluid (CSF) samples, volumetric and morphologic analysis using T1-weighted magnetic resonance imaging (MRI) and clinical assessments. Although the results from PET and CSF screening are promising, these interventions are more invasive than MRI. In the search for suitable MRI biomarkers, researchers have focused on characterizing early mild cognitive impairment (EMCI) and late mild cognitive impairment (LMCI) [6, 7]. Although the criteria for separating EMCI and LMCI are based on scores on memory tests, biomarkers in individuals with EMCI show a continuous spectrum to those in individuals with LMCI, implying that EMCI is a transitional stage of $\mathrm{AD}$ [8]. Thus, evaluating sequential changes in EMCI and LMCI should help in understanding early AD.

Diffusion tensor imaging (DTI) utilizes the diffusion of water molecules within tissues and provides axonal microstructural properties; thus, it is widely applied when studying white matter integrity $[9,10]$. Early AD studies using DTI have mainly focused on the white matter. However, since white matter changes in AD may be the result of Wallerian degeneration, followed by neurodegeneration in the gray matter $[11,12]$, the destruction of white matter is a less sensitive change in $\mathrm{AD}$.

The idea of measuring microstructural changes in gray matter using DTI has been demonstrated in both AD and frontotemporal dementia [13-15]. These studies showed that gray matter mean diffusivity (MD) is higher in patients than in healthy controls and that MD could be a promising imaging biomarker. However, there is a lasting notion that increased MD could be overestimated by the CSF signal, and this effect persists even with rigorous correction approaches, such as partial volume effects correction [16].

To overcome this problem, we adopted radiality, which presumably reflects the integrity of tangential cortical fibers. In an initial study of the applicability of radiality in $\mathrm{AD}$, we sought to find an association with conventional MRI biomarkers [17]. A recent study investigated the association between anisotropic diffusion and cortical structures through postmortem diffusion MRI along with histology in multiple sclerosis [18]. Although this study was limited to observing certain brain regions, it provides relevant evidence to measure cortical changes with DTI. Moreover, this parameter has been applied to study neurodevelopment and can distinguish the stages of aging [19-21]. Cortical microstructural changes are often observed with aging or neurodegeneration, which can be viewed as the opposite of neurodevelopment [22-25]. Thus, changes in fiber orientation may suggest cortical alterations and could be used as a biomarker in neurodegenerative diseases.

In this study, we hypothesized that the radiality within gray matter could be a microstructural measure of the cortex and used as the early signature of AD. We performed a cross-sectional surface-based cortical analysis approach using DTI, amyloid PET, and tau PET images to the AD continuum [17]. We evaluated whether gray matter radiality shows: i) early mesoscopic AD-related pathological change, and ii) complementarity with conventional $\mathrm{AD}$ biomarkers while providing distinct information regarding AD-related pathologies.

\section{Methods \\ Demographics}

Data used in this study were obtained from the Alzheimer's Disease Neuroimaging Initiative (ADNI) (adni.loni.usc.edu). The ADNI was launched in 2003 as a public-private partnership, led by Principal Investigator Michael W. Weiner, MD. The primary goal of ADNI has been to test whether serial magnetic resonance imaging (MRI), positron emission tomography (PET), other biological markers, and clinical and neuropsychological assessment can be combined to measure the progression of mild cognitive impairment $(\mathrm{MCI})$ and early Alzheimer's disease (AD). For up-to-date information, see www.adni-info.org.

From the ADNI database, we analyzed subjects who underwent both MRI and PET (amyloid, AV 45 and tau, AV 1451) including 78 cognitively normal $(\mathrm{CN}), 50$ EMCI, $34 \mathrm{LMCI}$, and $39 \mathrm{AD}$ individuals. Subjects were sampled according to the following criteria: age, around 60 to 90 years old, education, 12 to 20 years, and gendermatched within groups. To assess the $\mathrm{AD}$ continuum, amyloid-negative $\mathrm{CN}$ and amyloid-positive EMCI, LMCI, and AD subjects were selected. The EMCI group was subdivided into 38 dementia non-converters (stable EMCI) and 12 converters to assess changes in disease progression. A total of 201 subjects' T1 and DTI images were gathered from the ADNI. To increase the sample size, a multi-center approach was used, as discussed in [13]. The amyloid positivity of subjects was determined using whole brain PET AV45 standardized uptake value ratio (SUVR) with a 1.11 cutoff. Table 1 shows the demographics of the subjects used in this study; note 
Table 1 Demographics

\begin{tabular}{|c|c|c|c|c|c|c|c|}
\hline & $\mathrm{CN}(n=78)$ & $\mathrm{EMCl}(n=50)$ & $\begin{array}{l}\text { EMCI } \\
\text { Non-converter } \\
(n=38)\end{array}$ & $\begin{array}{l}\text { EMCI } \\
\text { Converter } \\
(n=12) \\
\end{array}$ & $\operatorname{LMCl}(n=34)$ & $A D(n=39)$ & Post hoc \\
\hline Female, n (\%) & $42(53.8)$ & $19(37.2)$ & $14(36.8)$ & $5(41.7)$ & $15(44.1)$ & $17(43.6)$ & - \\
\hline Age (SD) (y) & $72.7 \pm 5.9$ & $74.7 \pm 5.3$ & $74.1 \pm 4.9$ & $76.4 \pm 4.7$ & $73.9 \pm 5.6$ & $74.7 \pm 7.2$ & - \\
\hline Education (SD) (y) & $16.7 \pm 2.5$ & $15.2 \pm 2.6$ & $15.0 \pm 2.5$ & $15.6 \pm 3.1$ & $16.1 \pm 2.8$ & $15.4 \pm 2.9$ & - \\
\hline GCDR (SD) & 0.0 & 0.5 & 0.5 & 0.5 & 0.5 & $0.8 \pm 0.3$ & $C N<E M C l=L M C l<A D$ \\
\hline MMSE (SD) & $29.3 \pm 1.5$ & $28.2 \pm 1.2$ & $28.3 \pm 1.1$ & $28.1 \pm 1.7$ & $27.6 \pm 1.4$ & $24.4 \pm 4.0$ & $C N>E M C l=L M C l>A D$ \\
\hline MADAS-Cog (SD) & $9.7 \pm 6.8$ & $13.6 \pm 5.9$ & $13.2 \pm 5.0$ & $14.5 \pm 4.9$ & $14.6 \pm 4.8$ & $26.3 \pm 14.2$ & $C N<E M C l=L M C l<A D$ \\
\hline Immediate recall (SD) & $14.2 \pm 2.9$ & $10.4 \pm 3.4$ & $10.5 \pm 3.6$ & $9.9 \pm 2.7$ & $6.4 \pm 3.3$ & $3.8 \pm 2.0$ & $C N>E M C l>L M C l>A D$ \\
\hline Delayed recall (SD) & $12.8 \pm 3.4$ & $8.6 \pm 2.0$ & $8.6 \pm 2.1$ & $8.7 \pm 1.6$ & $3.1 \pm 2.7$ & $1.3 \pm 1.6$ & $C N>E M C l>L M C l>A D$ \\
\hline MRI center & $30 / 48$ & $40 / 10$ & $29 / 9$ & $11 / 1$ & $28 / 6$ & $36 / 3$ & - \\
\hline Florbetapir+, n (\%) & $0(0)$ & $50(100)$ & $38(100)$ & $12(100)$ & $34(100)$ & $39(100)$ & - \\
\hline AV1451 image, n (\%) & $44(68.8)$ & $9(14.1)$ & $8(21.1)$ & $1(8.33)$ & $5(7.8)$ & $3(4.7)$ & - \\
\hline
\end{tabular}

Data are $\mathrm{n}(\%)$ or mean \pm SD values. There were no gender, age, or year of education intergroup differences. GCDR, MMSE, and MADAS-Cog Scores in EMCI and $\mathrm{LMCl}$ did not show significant differences. Analysis of variance with Tukey test was used for post hoc analysis with $p<0.05$. For MRI data, two major scanners were used: GE and SIEMENS and delineated as MRI center GE/SIEMENS

$A D$ Alzheimer's disease, CN Cognitively normal, EMCI Early mild cognitive impairment, GCDR Global Clinical Dementia Rating, LMCI Late mild cognitive impairment, MADAS-Cog Modified Alzheimer's Disease Assessment Scale-Cognitive subscale, MMSE Mini Mental State Examination

that $44 \mathrm{CN}$ subjects, nine EMCI subjects, five LMCI subjects, and three AD subjects underwent AV1451 tau PET imaging. An additional $28 \mathrm{CN}$ subjects with amyloid positivity were analyzed to identify the earliest $A D$ pathological changes as presented in Supp. Table 1.

\section{Image processing}

T1-weighted images were processed using FreeSurfer package v6.0 (http://surfer.nmr.mgh.harvard.edu) as previously reported in [13]. Cortical thickness (CTh) maps were registered to the FreeSurfer average sphere using spherical registration for group comparison. DTI and PET images were registered with respect to T1 images using a boundary-based algorithm for further processing. DTI images were processed using the FSL package as follows: eddy current correction, rotate gradient vectors from the results of eddy correction, and tensor fitting to produce the MD map and primary eigenvector map. DTI metrics were further processed to avoid partial volume effects following Koo et al. [26]. PET images were partial volume corrected using mri_gtmpvc which is built into the FreeSurfer package. PET images were normalized by mean signal from the whole cerebellum and converted to SUVR for amyloid and tau PET, AV45, and AV1451, respectively. The images were then boundarybased registered to corresponding $\mathrm{T} 1$ structural images. To avoid any partial volume effects, the center parts of the cortical column were sampled for surface analysis. Lastly, CTh was smoothed with a 10-mm full width half maximum Gaussian kernel, while other modalities were smoothed with a 15-mm kernel. Figure 1 shows an overall schematic of the process.

\section{Calculation of radiality}

A surface normal vector was obtained from the individual gray matter surface to define the cortical orientation. FreeSurfer represents the surface in triangular meshes, and the surface normal vector can be computed using the cross-product between edges. The vertex-wise dot product between the primary diffusion direction, primary eigenvector of the diffusion tensor, and the surface normal vector was quantified as a radiality index, $r$, where $v$ represents the surface normal vector and $e_{1}$ represents the primary diffusion direction [22].

$$
r=\left|\hat{v}_{n} \cdot \hat{e}_{1}\right|
$$

It ranges from 0 to 1 , where $r=0$ indicates tangential diffusion and $r=1$ indicates radial diffusion to the cortex. The subject's principal eigenvector map was projected onto the individual surface reconstruction to calculate vertex-wise radiality, as discussed in [22].

\section{Cutoff analysis}

To further test the feasibility of radiality as an AD biomarker, we performed cutoff analysis using receiver operating characteristic graphs to distinguish $\mathrm{CN}$ from different AD stages, as shown in Supp. Table 2. The feature used was the mean radiality within the cluster obtained from the $\mathrm{CN}$ vs. EMCI group comparison. By 


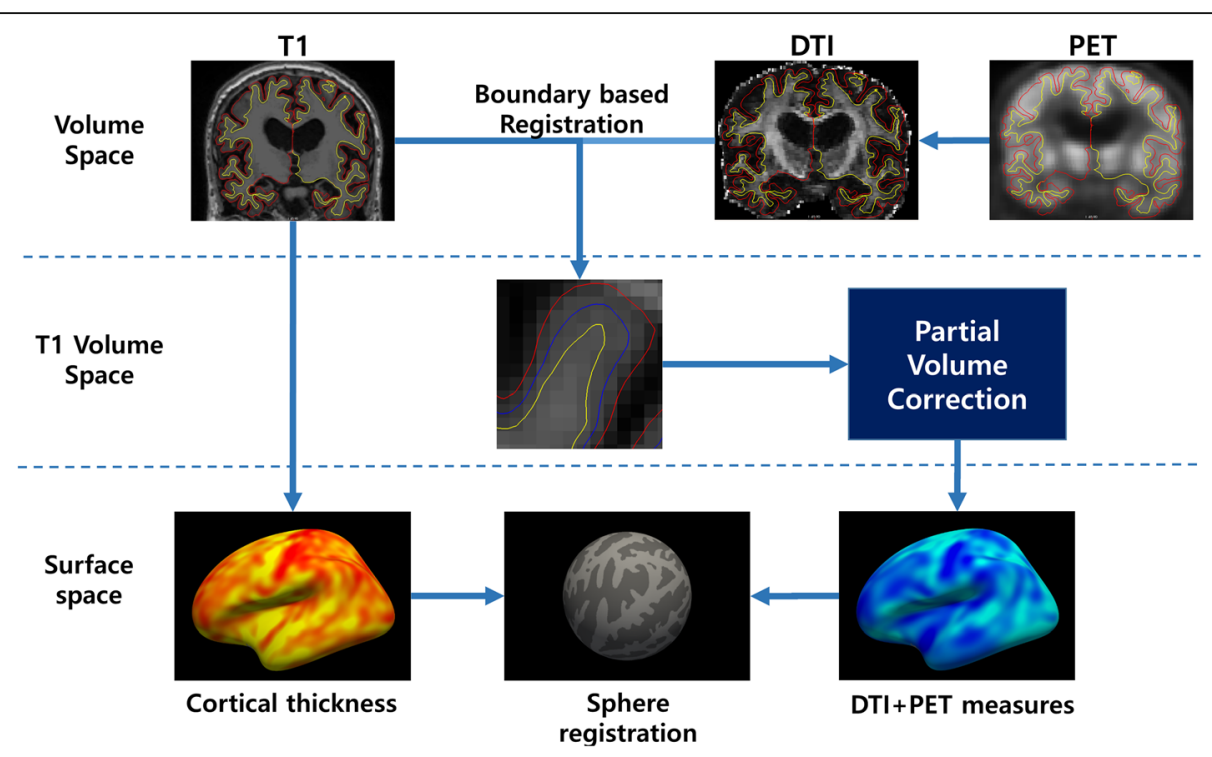

Fig. 1 Overall scheme for surface projection analysis. DTI and PET images were boundary-based registered to the T1 image and projected to the fsaverage surface for group comparison

varying the cutoff, we sought to find the cost-effective point where it minimizes the difference between sensitivity and specificity [27].

\section{Statistical analysis}

We first compared the differences between groups for radiality, CTh, MD, AV45, and AV1451 using a general linear model, which is available in FreeSurfer. The results were cluster-wise corrected for a family-wise error (FWE)-corrected $p$-value $<0.05$.

To test the associations between radiality and other neuroimaging biomarkers, we calculated a set of vertexwise partial correlations with radiality as the dependent variable and CTh, MD, AV45, and AV1451 as the independent variable. Age, gender, years of education, and MRI center were set as covariates for cluster analyses. A permutation test was applied to account for multiple comparisons using a Monte Carlo simulation with 10, 000 repeats, which is a built-in function of FreeSurfer.

To test the linear relationship between radiality and other neuroimaging biomarkers, we quantified mean metrics within AD-specific ROIs. ROIs include the entorhinal, fusiform, insula, inferior, middle, and superior temporal cortex. Mean metrics within ROIs were plotted in box and whisker plots and are presented in Fig. 5e and Fig. 6. Significant differences between groups were tested using one-way analysis of variance (ANOVA).

\section{Results}

Group comparison along $A D$ continuum

We first compared radiality, CTh, and MD differences between groups, i.e., $\mathrm{CN}$ vs. $\mathrm{EMCI}, \mathrm{CN}$ vs. $\mathrm{LMCI}$, and
$\mathrm{CN}$ vs. $\mathrm{AD}$. The results were cluster-wise corrected for a FWE-corrected $p<0.05$. Figure 2 shows the significant group different clusters ranging from a $p$-value of 0.05 to $10^{-5}$. Only radiality could delineate the difference between EMCI and $\mathrm{CN}$. Compared to $\mathrm{CN}$, all groups showed decreased radiality, decreased $\mathrm{CTh}$, and increased MD. There was no group difference in radiality between EMCI and LMCI.

\section{EMCI non-converter versus converter}

We compared radiality between $\mathrm{CN}$ and EMCI nonconverter, $\mathrm{CN}$ and EMCI converter, and EMCI nonconverter vs. converter. The results were cluster-wise corrected for a FWE-corrected $p<0.05$. Figure 3 shows significant group different clusters ranging from a $p$ value of 0.05 to $10^{-5}$. Compared to $\mathrm{CN}$, the EMCI nonconverter showed decreased radiality in the left superior frontal and superior parietal cortices. The EMCI converter showed decreased radiality mainly in the bilateral insula cortex. Direct comparison between EMCI nonconverter and converter delineated the bilateral insular, left superior frontal, and right precentral cortex.

\section{Partial correlation between radiality and other imaging biomarkers}

We then found a vertex-wise correlation between radiality and other imaging biomarkers, as shown in Fig. 4. CTh showed mostly positive correlations with decrease in cortical thickness accompanied by a decrease in radiality. MD mostly negatively correlated with an increase in MD accompanied by a decrease in radiality. Amyloid and tau levels negatively correlated with radiality. 


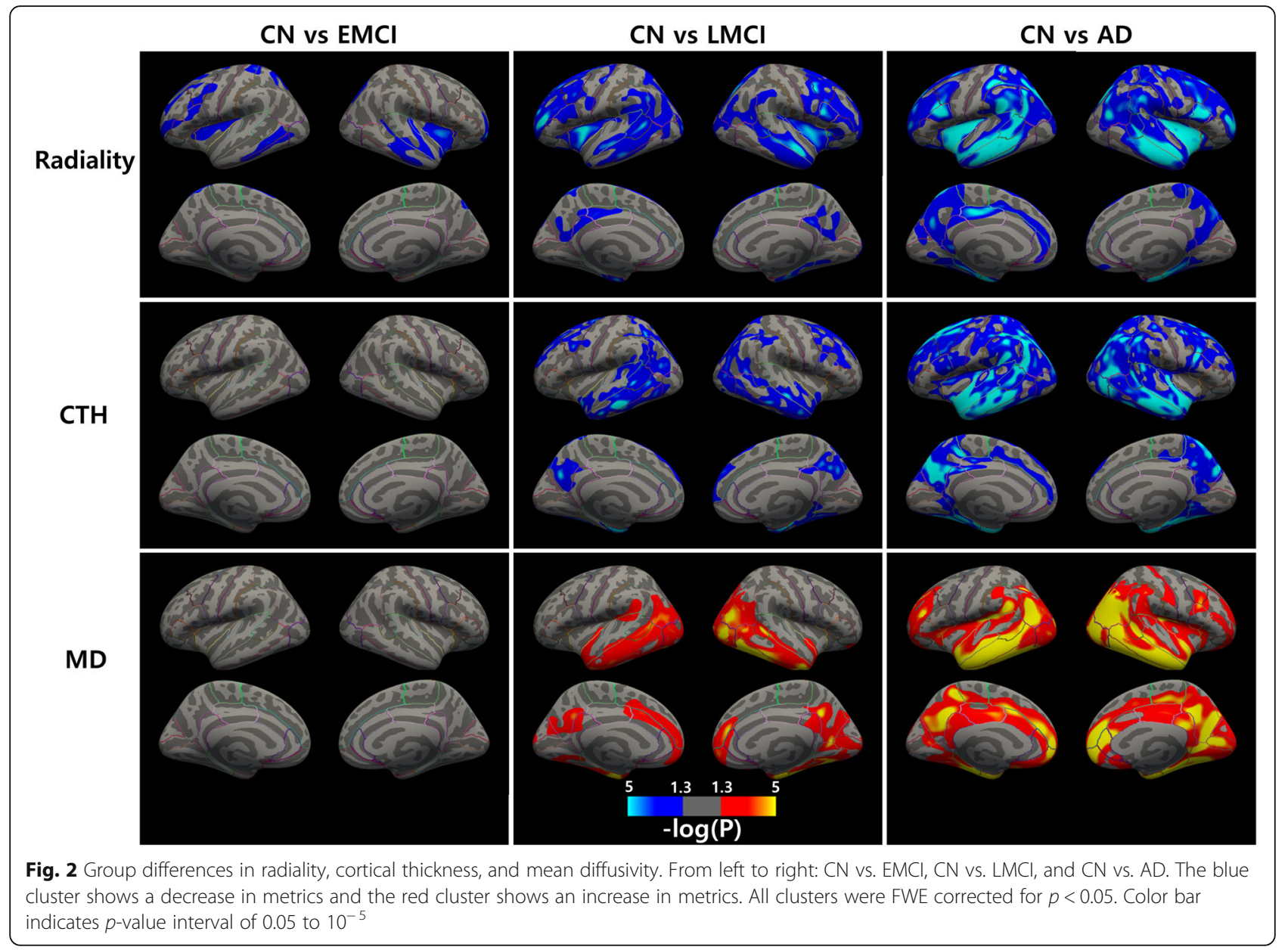

\section{Correlations between radiality and other imaging} biomarkers

In order to find progressive changes in radiality reflective of disease progression, an $\mathrm{AD}$-specific $\mathrm{ROI}$ mask was used to calculate mean biomarker data. Each subject's mean data were scatter-plotted and used to calculate the Pearson correlation, as shown in Fig. 5. The correlation for CTh was $R=0.641$, that for MD was $R=-0.677$, that for AV45 was $R=-0.490$, and that for AV1451 was $R=$ -0.412 with radiality.

\section{Radiality dynamics from AD-specific ROIs}

To find generative changes in radiality during disease progression, the mean radiality in $\mathrm{AD}$ ROIs was calculated for direct comparison between groups. Radiality within AD-specific ROIs was plotted in a box and

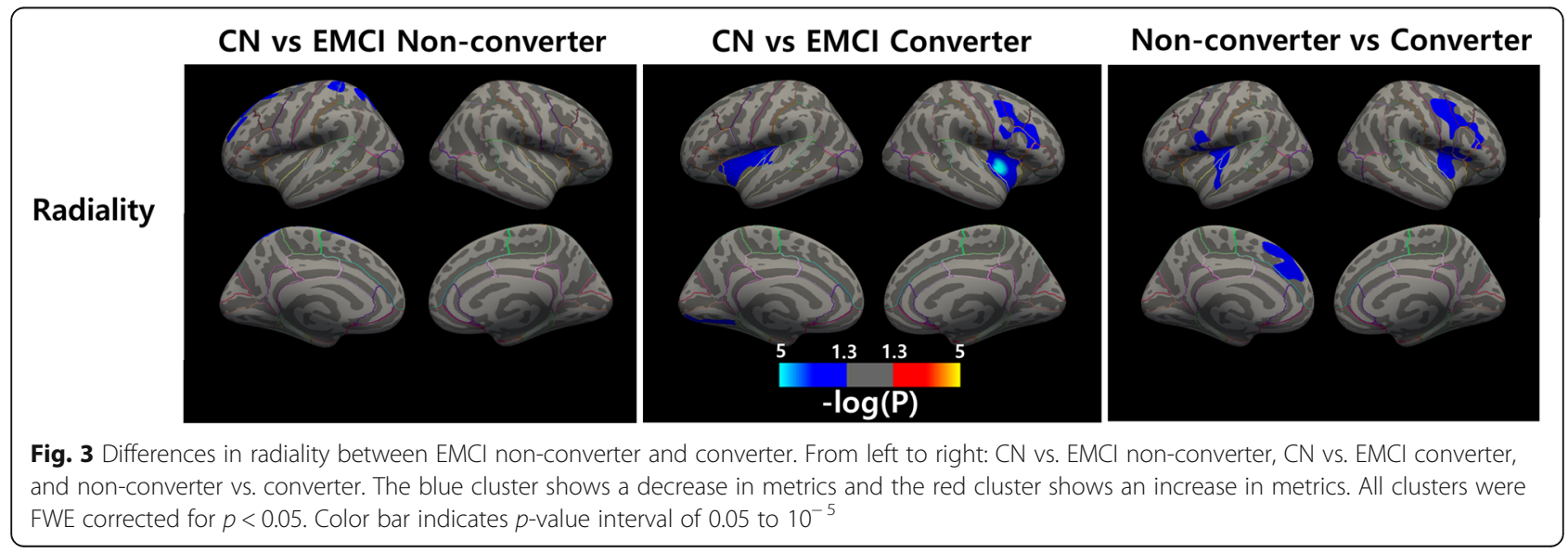




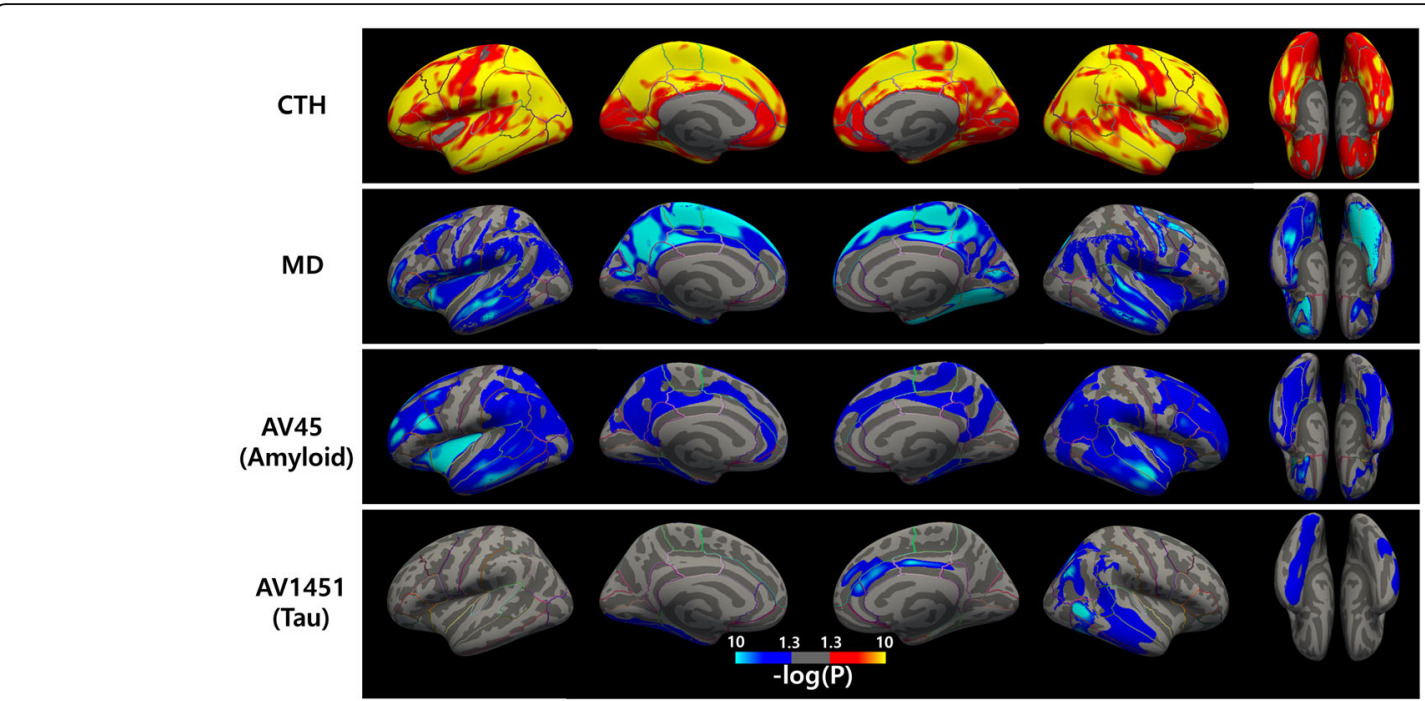

Fig. 4 Partial correlation between radiality and image biomarkers. The red cluster shows a positive correlation with radiality, and the blue cluster shows a negative correlation. Cortical thickness showed positive correlations, and mean diffusivity, AV45, and AV1451 showed negative correlations. Color bar indicates $p$-value interval of 0.05 to $10^{-10}$

whisker plot, as shown in Fig. 6. The results showed decreasing radiality with disease progression. Significance was tested with one-way ANOVA with $p$-values $<0.05$, 0.01 , and 0.001 . The insula, middle, and superior temporal cortex showed the most radiality reduction with disease progression.

\section{Cutoff analysis using radiality}

Classification of $\mathrm{CN}$ vs. EMCI showed $70.5 \%$ accuracy with $70.2 \%$ sensitivity, $72.7 \%$ specificity, and 0.766 AUC. Subsequent analysis to distinguish between $\mathrm{CN}$ and LMCI, MCI group (EMCI+LMCI), $\mathrm{AD}$, and patient group $(\mathrm{EMCI}+\mathrm{LMCI}+\mathrm{AD})$ also showed similar results to those presented in Supp. Table 2.

\section{Discussion}

In this study, we investigated the early features of EMCI using cortical radiality, which reflects mesoscopic structural changes. By leveraging radiality in the gray matter, we could detect the changes in EMCI that were not detected using conventional MRI biomarkers. We found progressively larger regions of decreased radiality as the disease progressed, starting from the medial temporal cortex in EMCI to the whole brain in AD. However, CTh or MD did not show significant differences between $\mathrm{CN}$ and EMCI. Furthermore, the radiality results from $\mathrm{CN}$ and EMCI non-converter showed similar patterns to those of $\mathrm{CN}$ amyloid-negative and -positive (Supp. Figure 1). Based on our results, the microstructural gray matter changes in the bilateral insular cortex are associated with disease progression as seen in the $\mathrm{CN}$ and EMCI converter results.

We investigated the relationship between radiality and other imaging measures. The association between radiality and CTh showed a strong positive correlation in various regions of the brain, as shown in Fig. 4. It is clear that a higher CTh indicates a deeper cortical structure, and fiber orientation tends to have a radial orientation. Cortical depth profile analysis showed that thicker cortex was related to larger radiality [28]. In addition, MD showed a strong negative correlation in the temporal, parietal, and frontal cortices. Radiality may be sensitive to CTh but also reflects microstructural features. With AV45 and AV1451, radiality showed an association that widely overlapped with both CTh and MD. Thus, radiality may also reflect changes due to pathologic protein accumulation within the cortex.

Although microstructural changes associated with radiality are unclear, one plausible feature is the disorganization of tangential cortical fibers. It has been reported that tangential cortical fibers develop during neurodevelopment and aging [22]. There are several events that lead to an increase in tangentially oriented fibers, including dendritic elaboration [29], formation of local circuits [30], expansion of thalamo-cortical fibers [31], and disappearance of radial glia [32, 33]. A decrease in radiality may be contrary to those of neurodevelopment. For instance, synaptic loss, neuronal soma changes and neurite disorganization, along with neuronal loss, may lead to a decrease in radiality. These changes may be concurrent with the net loss of macromolecules that affect diffusivity, increasing free water in the extracellular space. However, radiality provides evidence of neuronal density that explains concurrent cortical atrophy. Furthermore, accumulation of amyloid or tau proteins may also participate in the disruption of the microstructure. Given that radiality can identify EMCI, we can 


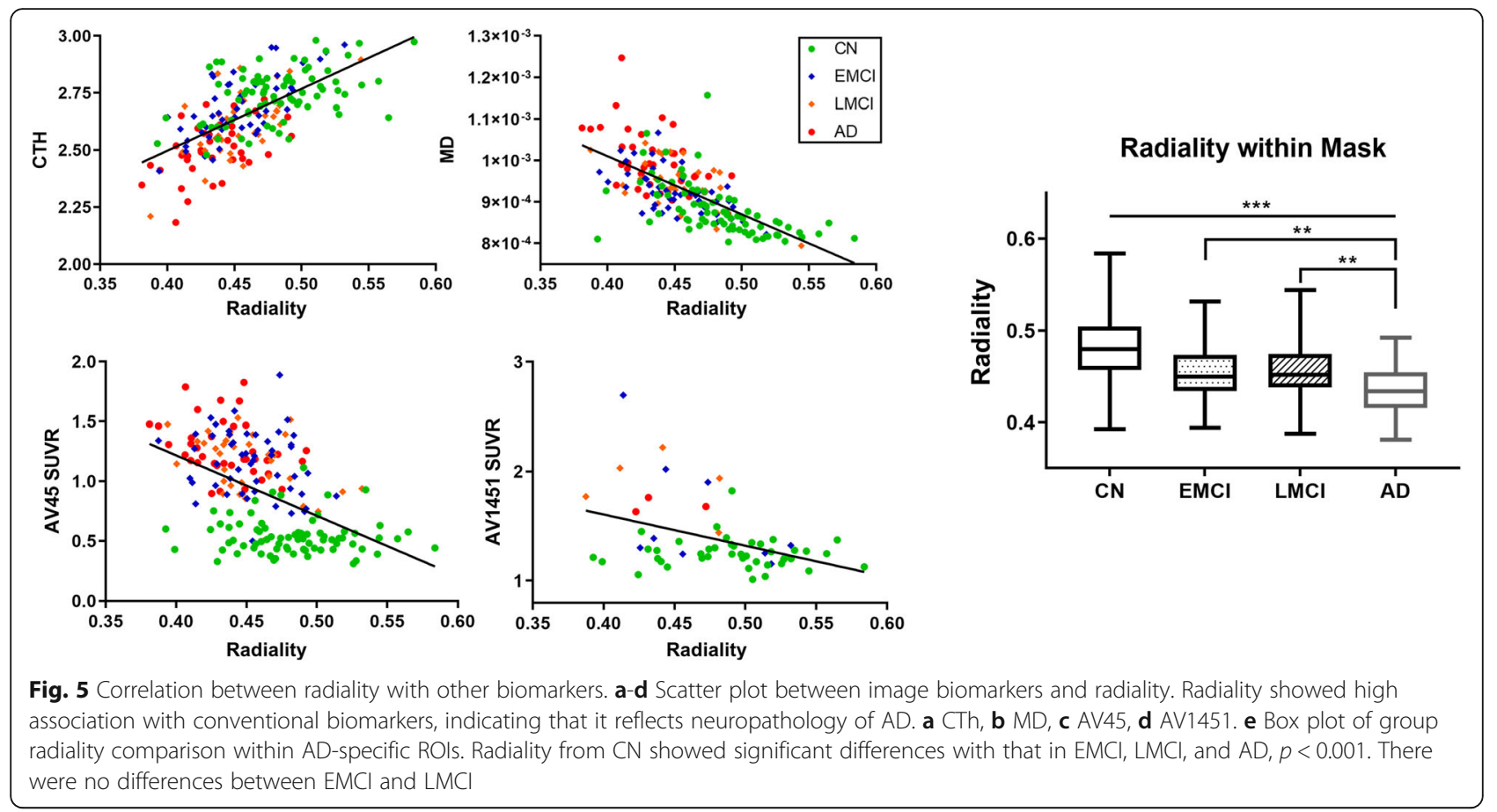

further speculate that these microstructural changes occur in the earlier stages of $\mathrm{AD}$, which are not apparent in macroscopic investigation.

To test the sensitivity of radiality, we sought to identify the earliest stage of AD. Interestingly, our $\mathrm{CN}$ vs. EMCI radiality analysis did not show a biphasic trajectory, as discussed in a previous work [34]. Thus, we conducted an additional analysis on amyloid-negative $\mathrm{CN}$ and amyloid-positive CN (Supp Fig. 1). We observed biphasic behavior for CTh and MD, where biomarkers showed opposite directions of changes. While CTh increased and MD decreased, radiality showed a monotonous

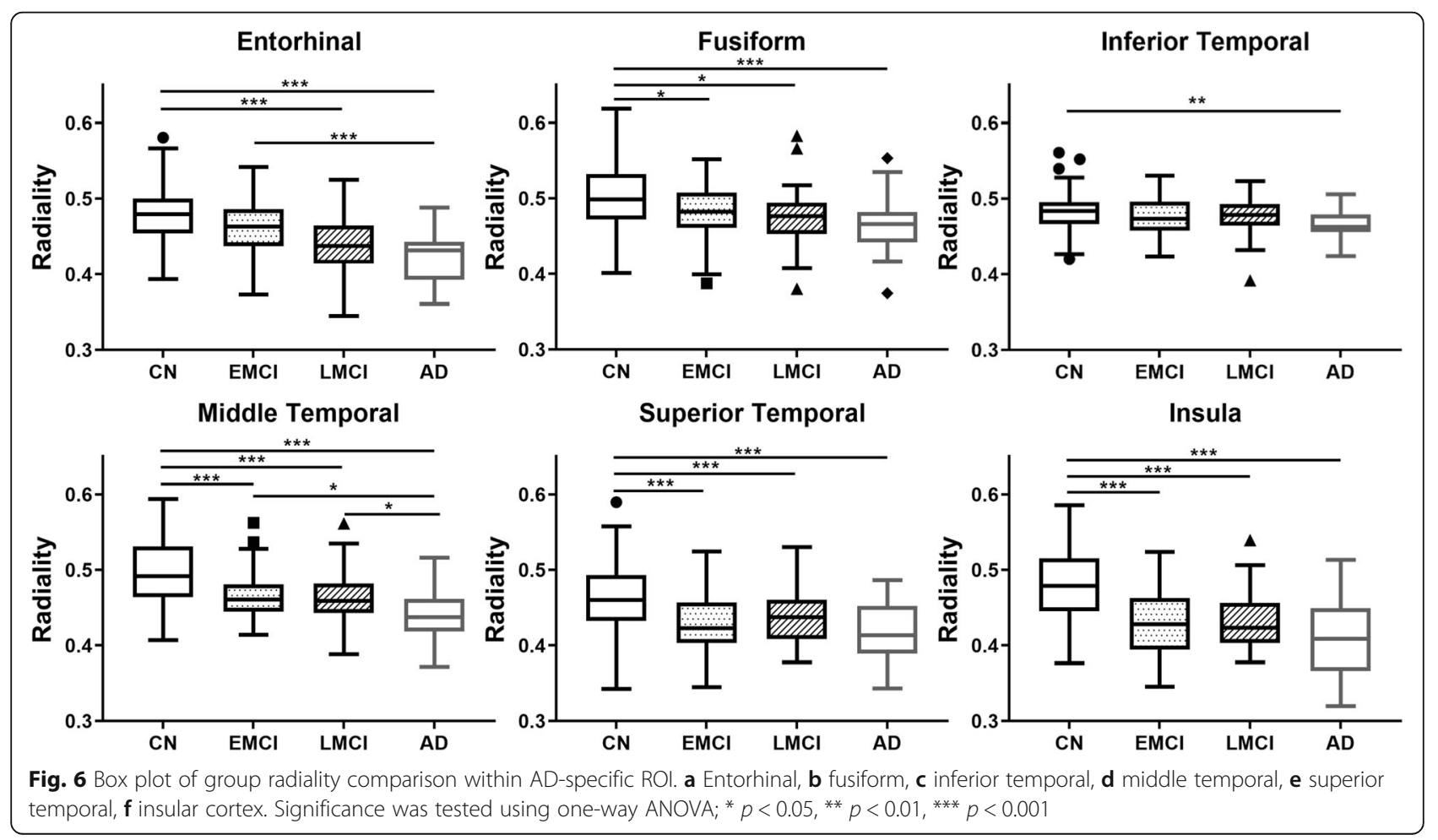


decrease in amyloid-positive $\mathrm{CN}$. This distinct behavior of radiality could characterize the changes in EMCI, while CTh and MD could not. Both the CTh increase and $\mathrm{MD}$ decrease in the early stage of $\mathrm{AD}$ were thought to be caused by an amyloid-induced inflammatory response [13]. However, radiality seems to decrease whenever there are microstructural changes in the tissue. In a preterm study, the occipital cortex showed a decrease in radiality as in early development [19-21]. In the case of multiple sclerosis, decreased radiality was observed in the dorsolateral prefrontal cortex, Heschl's gyrus, and primary visual cortex, possibly due to cortical alterations [18].

We performed a simple cutoff binary classification analysis to assess the diagnostic accuracy of radiality. The target mask was obtained from the group comparison result of $\mathrm{CN}$ versus $\mathrm{EMCI}$, and the individual mean radiality within the mask was used as a classification feature. With varying cutoff values, the model showed $70.5 \%$ accuracy with 0.766 AUC to differentiate $\mathrm{CN}$ and EMCI, $67.9 \%$ with 0.757 AUC for CN and LMCI, 70.5\% with $0.766 \mathrm{AUC}$ for $\mathrm{CN}$ and $\mathrm{MCI}$ (EMCI+LMCI), and $78.6 \%$ with 0.867 AUC to $\mathrm{CN}$ and $\mathrm{AD}$, as presented in Supp. Table 2. The results were comparable to those of previous studies. A recent study that adopted a logistic regression model with neurite density index, orientation dispersion index, and CTh as features reported 0.72 AUC for $\mathrm{CN}$ and $\mathrm{MCI}$ and $0.91 \mathrm{AUC}$ for $\mathrm{CN}$ and $\mathrm{AD}$ [35]. Other studies employing whole MD and gray matter map reported $79.6 \%$ accuracy with 0.84 AUC for $\mathrm{CN}$ and $\mathrm{MCI}$ and $93.5 \%$ with $0.94 \mathrm{AUC}$ to $\mathrm{CN}$ and $\mathrm{AD}$ [36], $76 \%$ with 0.83 AUC for NC and AD [37].

There were several limitations to the current study. First, the relatively poor resolution of DTI compared to structural T1 images could lead to inaccurate results. Although surface analysis was employed to mitigate registration or segregation errors, a higher resolution DTI would be needed to observe precise cortical changes. Second, the use of multi-protocol DTI images could influence the observation of progressive changes in MCI. We sought to control for age, gender, years of education, and MRI center between the group while applying harmonization to minimize the variation between subjects [38]. Third, the number of subjects who underwent tau PET imaging was not enough to identify any relationship with tau pathology. In order to focus on progressive changes, not only showing a relationship with amyloid but also with tau is an important aspect [39]. However, several subjects in this study underwent screening only once without follow-up or only MRI data were available.

\section{Conclusions}

In conclusion, we investigated the cortical changes in EMCI using structural MRI, DTI, and PET imaging markers. Only radiality could delineate the changes in EMCI while cortical thickness and MD could not. In addition, radiality changes in the frontal cortex as well as amyloid deposits in the continuum. These results indicate that the multimodal approach, atrophy and microstructure, may illuminate early changes in $\mathrm{AD}$. However, further studies are needed to support the relationship between alterations in cortical structure and diffusion orientation.

\section{Supplementary information}

Supplementary information accompanies this paper at https://doi.org/10. 1186/s12883-020-01939-2.

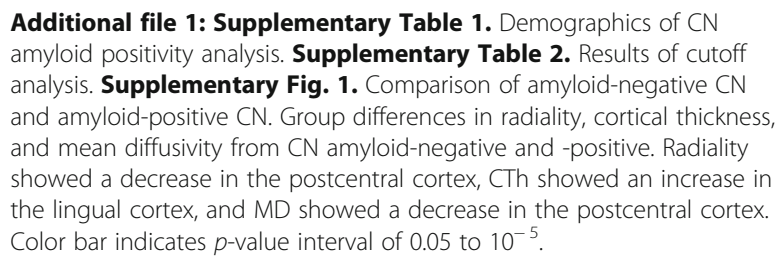

\section{Abbreviations}

AD: Alzheimer's disease; ADNI: Alzheimer's Disease Neuroimaging Initiative; CN: Cognitively normal; CSF: Cerebrospinal fluid; CTh: Cortical thickness; DTI: Diffusion tensor imaging; EMCl: Early mild cognitive impairment; GCDR: Global clinical dementia ratings; LMCl: Late mild cognitive impairment; MADAS-Cog: Modified Alzheimer's disease Assessment Scale cognitive subscale; MD: Mean diffusivity; MMSE: Mini Mental State Examination; MRI: Magnetic resonance imaging; PET: Positron emission tomography; ROI: Region of interest; SUVR: Standardized uptake value ratio

\section{Acknowledgements}

Data collection and designing for this project was funded by the Alzheimer's Disease Neuroimaging Initiative (ADNI) (National Institutes of Health Grant U01 AG024904) and DOD ADNI (Department of Defense award number W81XWH-12-2-0012). ADNI is funded by the National Institute on Aging, the National Institute of Biomedical Imaging and Bioengineering, and through generous contributions from the following: AbbVie, Alzheimer's Association; Alzheimer's Drug Discovery Foundation; Araclon Biotech; BioClinica, Inc.; Biogen; Bristol-Myers Squibb Company; CereSpir, Inc.; Eisai Inc.; Elan Pharmaceuticals, Inc.; Eli Lilly and Company; Eurolmmun; F. Hoffmann-La Roche Ltd. and its affiliated company Genentech, Inc.; Fujirebio; GE Healthcare; IXICO Ltd.; Janssen Alzheimer Immunotherapy Research \& Development, LLC.; Johnson \& Johnson Pharmaceutical Research \& Development LLC.; Lumosity; Lundbeck; Merck \& Co., Inc.; Meso Scale Diagnostics, LLC.; NeuroRx Research; Neurotrack Technologies; Novartis Pharmaceuticals Corporation; Pfizer Inc.; Piramal Imaging; Servier; Takeda Pharmaceutical Company; and Transition Therapeutics. The Canadian Institutes of Health Research is providing funds to support ADNI clinical sites in Canada. Private sector contributions are facilitated by the Foundation for the National Institutes of Health (www.fnih.org). The grantee organization is the Northern California Institute for Research and Education, and the study is coordinated by the Alzheimer's Disease Cooperative Study at the University of California, San Diego. ADNI data are disseminated by the Laboratory for Neuro Imaging at the University of Southern California.

Data used in preparation of this article were obtained from the Alzheimer's Disease Neuroimaging Initiative (ADNI) database (adni.loni.usc.edu). As such, the investigators within the ADNI contributed to the design and implementation of ADNI and/or provided data but did not participate in analysis or writing of this report. A complete listing of ADNI investigators can be found at: http://adni.loni.usc.edu/wp-content/uploads/how_to_apply/ ADNI_Acknowledgement_List.pdf

Authors' contributions

$\mathrm{PL}, \mathrm{YJ}$, and HK contributed to the study conception and design. Material preparation, data collection and analysis were performed by PL. The first draft of the manuscript was written by PL. YJ and HK commented on 
previous versions of the manuscript. The authors read and approved the final manuscript. Data used in preparation of this article were obtained from the ADNI database. As such, the investigators within the ADNI contributed to the design and implementation of ADNI and/or provided data but did not participate in analysis or writing of this report.

\section{Funding}

This work was supported in part by grant HI14C2768 from the Korea Health Technology Research and Development Project through the Korea Health Industry Development Institute, funded by the Ministry of Health \& Welfare, Republic of Korea, and in part by the Bio \& Medical Technology Development Program (2016941946) through the National Research Foundation of Korea (NRF), funded by the Ministry of Science, ICT and Future Planning. All these funds provided computing resources for this study but did not participate in the design of the study and collection, analysis and interpretation of data in writing the manuscript.

\section{Availability of data and materials}

The MRI and PET data were downloaded from the Alzheimer's Disease Neuroimaging Initiative (ADNI) database (http://adni.loni.usc.edu/). Application for access to the ADNI data can be submitted by anyone at http://adni.loni.usc.edu/data-samples/access-data/. The process includes completion of an online application form and acceptance of Data Use Agreement.

\section{Ethics approval and consent to participate}

The institutional review boards of all participating ADNI sites reviewed and approved the data collection protocol provided by ADNI. List of ethics committee can be found in the supplementary information. For up-to-date information, see www.adni-info.org. Upon accessing the database, we have received administrative approval for access to the ADNI database.

\section{Consent for publication}

Not applicable.

\section{Competing interests}

The authors declare that they have no competing interests.

\section{Author details}

'Department of Bio and Brain Engineering, Korea Advanced Institute of Science and Technology, Daehak-ro 291, Yuseong-gu, Daejeon 34141, Republic of Korea. ${ }^{2} \mathrm{KI}$ for Health Science and Technology, Korea Advanced Institute of Science and Technology, Daejeon, Republic of Korea. ${ }^{3}$ Graduate School of Medical Science and Engineering, Korea Advanced Institute of Science and Technology, Daejeon, Republic of Korea.

Received: 10 February 2020 Accepted: 23 September 2020

Published online: 02 October 2020

\section{References}

1. Jack CR Jr, Knopman DS, Jagust WJ, Shaw LM, Aisen PS, Weiner MW, et al. Hypothetical model of dynamic biomarkers of the Alzheimer's pathological cascade. Lancet Neurol. 2010;9(1):119-28. https://doi.org/10.1016/S14744422(09)70299-6 PMID 20083042.

2. Shim YS, Morris JC. Biomarkers predicting Alzheimer's disease in cognitively normal aging. J Clin Neurol. 2011;7(2):60-8. https://doi.org/10.3988/jcn.2011. 7.2.60 PMID 21779293.

3. Cummings JL, Morstorf T, Zhong K. Alzheimer's disease drug-development pipeline: few candidates, frequent failures. Alzheimers Res Ther. 2014;6(4):37. https://doi.org/10.1186/alzrt269 PMID 25024750.

4. Anderson RM, Hadjichrysanthou C, Evans S, Wong MM. Why do so many clinical trials of therapies for Alzheimer's disease fail? Lancet. 2017; 390(10110):2327-9. https://doi.org/10.1016/S0140-6736(17)32399-1 PMID 29185425.

5. Schott JM, Aisen PS, Cummings JL, Howard RJ, Fox NC. Unsuccessful trials of therapies for Alzheimer's disease. Lancet. 2019;393(10166):29. https://doi.org/ 10.1016/S0140-6736(18)31896-8 PMID 30614456.

6. Aisen PS, Petersen RC, Donohue MC, Gamst A, Raman R, Thomas RG, et al. Clinical core of the Alzheimer's disease neuroimaging initiative: progress and plans. Alzheimers Dement. 2010;6(3):239-46. https://doi.org/10.1016/j. jalz.2010.03.006 PMID 20451872.
7. Weiner MW, Aisen PS, Jack CR Jr, Jagust WJ, Trojanowski JQ, Shaw L, et al. The Alzheimer's Disease Neuroimaging Initiative: progress re-port and future plans. Alzheimers Dement. 2010;6(3):202-11.e7. https://doi.org/10.1016/j.jalz. 2010.03.007 PMID 20451868.

8. Qiu Y, Li L, Zhou TY, Lu W, Alzheimer's Disease Neuroimaging Initiative. Alzheimer's disease progression model based on integrated biomarkers and clinical measures. Acta Pharmacol Sin. 2014;35(9):1111-20. https://doi.org/10. 1038/aps.2014.57 PMID 25088003.

9. Basser PJ, Mattiello J, LeBihan D. MR diffusion tensor spectroscopy and imaging. Biophys J. 1994;66(1):259-67. https://doi.org/10.1016/S00063495(94)80775-1 PMID 8130344.

10. Pierpaoli C, Basser PJ. Toward a quantitative assessment of diffusion anisotropy. Magn Reson Med. 1996;36(6):893-906. https://doi.org/10.1002/ mrm.1910360612 PMID 8946355.

11. Bozzali M, Cercignani M, Sormani MP, Comi G, Filippi M. Quantification of brain gray matter damage in different ms phenotypes by use of diffusion tensor MR imaging. AJNR Am J Neuroradiol. 2002;23(6):985-8 PMID 12063230.

12. Coleman MP, Freeman MR. Wallerian degeneration, wld(s), and nmnat. Annu Rev Neurosci. 2010;33:245-67. https://doi.org/10.1146/annurev-neuro060909-153248 PMID 20345246.

13. Montal V, Vilaplana E, Alcolea D, Pegueroles J, Pasternak O, González-Ortiz S, et al. Cortical microstructural changes along the Alzheimer's disease continuum. Alzheimers Dement. 2018;14(3):340-51. https://doi.org/10.1016/j. jalz.2017.09.013 PMID 29080407.

14. Illán-Gala I, Montal V, Borrego-Écija S, et al. Cortical microstructure in the behavioural variant of frontotemporal dementia: looking beyond atrophy. Brain. 2019;142(4):1121-33. https://doi.org/10.1093/brain/awz031 PMID 30906945.

15. Lee P, Ryoo H, Park J, Jeong Y, Alzheimer's Disease Neuroimaging Initiative. Morphological and microstructural changes of the hippocampus in early MCl: a study utilizing the Alzheimer's Disease Neuroimaging Initiative database. J Clin Neurol. 2017;13(2):144-54. https://doi.org/10.3988/jcn.2017. 13.2.144 PMID 28176504

16. Henf J, Grothe MJ, Brueggen K, Teipel S, Dyrba M. Mean diffusivity in cortical gray matter in Alzheimer's disease: the importance of partial volume correction. Neurolmage Clin. 2018;17:579-86. https://doi.org/10.1016/j.nicl. 2017.10.005 PMID 29201644.

17. Lee $\mathrm{P}, \mathrm{Kim} H \mathrm{HR}$, Jeong $\mathrm{Y}$. Disruption of gray matter microstructure in Alzheimer's disease continuum using fiber orientation international conference of Korean dementia association. Vol. 133; 2019. p. FP-0007.

18. McKavanagh R, Torso M, Jenkinson M, Kolasinski J, Stagg CJ, Esiri MM, et al. Relating diffusion tensor imaging measurements to microstructural quantities in the cerebral cortex in multiple sclerosis. Hum Brain Mapp. 2019:40(15):4417-31. https://doi.org/10.1002/hbm.24711 PMID 31355989.

19. Eaton-Rosen Z, Scherrer B, Melbourne A, Ourselin S, Neil JJ, Warfield SK. Investigating the maturation of microstructure and radial orientation in the preterm human cortex with diffusion MRI. Neuroimage. 2017;162:65-72. https://doi.org/10.1016/..neuroimage.2017.08.013 PMID 28801253.

20. Kroenke CD. Using diffusion anisotropy to study cerebral cortical gray matter development. J Magn Reson. 2018;292:106-16. https://doi.org/10. 1016/j.jmr.2018.04.011 PMID 29705039.

21. Dean DC III, O'Muircheartaigh J, Dirks H, Travers BG, Adluru N, Alexander AL, et al. Mapping an index of the myelin g-ratio in infants using magnetic resonance imaging. Neuroimage. 2016;132:225-37. https://doi.org/10.1016/j. neuroimage.2016.02.040 PMID 26908314.

22. McNab JA, Polimeni JR, Wang R, Augustinack JC, Fujimoto K, Stevens A, et al. Surface based analysis of diffusion orientation for identifying architectonic domains in the in vivo human cortex. Neurolmage. 2013;69: 87-100. https://doi.org/10.1016/j.neuroimage.2012.11.065 PMID 23247190.

23. Fukutomi H, Glasser MF, Zhang H, Autio JA, Coalson TS, Okada T, et al. Neurite imaging reveals microstructural variations in the human cerebral cortical gray matter. Neuroimage. 2018;182:488-99. https://doi.org/10.1016/j. neuroimage.2018.02.017 PMID 29448073.

24. Wu D, Reisinger D, Xu J, Fatemi SA, van Zijl PC, Mori S, et al. Localized diffusion magnetic resonance micro-imaging of the live mouse brain. Neuroimage. 2014;91:12-20. https://doi.org/10.1016/j.neuroimage.2014.01. 014 PMID 24440780.

25. Szczepankiewicz F, Lasič S, van Westen D, Sundgren PC, Englund E, Westin $C F$, et al. Quantification of microscopic diffusion anisotropy disentangles effects of orientation dispersion from microstructure: applications in healthy 
volunteers and in brain tumors. Neuroimage. 2015;104:241-52. https://doi. org/10.1016/j.neuroimage.2014.09.057 PMID 25284306.

26. Koo BB, Hua N, Choi CH, Ronen I, Lee JM, Kim DS. A framework to analyze partial volume effect on gray matter mean diffusivity measurements. Neuroimage. 2009;44(1):136-44. https://doi.org/10.1016/j.neuroimage.2008. 07.064 PMID 18775785

27. Cardillo G. ROC curve: compute a receiver operating characteristics curve. Github; 2020. Available from: https://github.com/dnafinder/roc.

28. Truong TK, Guidon A, Song AW. Cortical depth dependence of the diffusion anisotropy in the human cortical gray matter in vivo. PLoS One. 2014;9(3): e91424. https://doi.org/10.1371/journal.pone.0091424 PMID 24608869.

29. Marín-Padilla M. Ontogenesis of the pyramidal cell of the mammalian neocortex and developmental cytoarchitectonics: a unifying theory. J Comp Neurol. 1992;321(2):223-40. https://doi.org/10.1002/cne.903210205 PMID 1500541.

30. Callaway EM, Katz LC. Emergence and refinement of clustered horizontal connections in cat striate cortex. J Neurosci. 1990;10(4):1134-53. https://doi. org/10.1523/JNEUROSCI.10-04-01134.1990 PMID 2329372.

31. Ghosh A, Shatz CJ. A role for subplate neurons in the patterning of connections from thalamus to neocortex. Development. 1993;117(3):103147 PMID 8325233.

32. Rivkin MJ, Flax J, Mozell R, Osathanondh R, Volpe JJ, Villa-Komaroff L, Oligodendroglial development in human fetal cerebrum. Ann Neurol. 1995; 38(1):92-101. https://doi.org/10.1002/ana.410380116 PMID 7611731.

33. Hardy RJ, Friedrich VL Jr. Oligodendrocyte progenitors are generated throughout the embryonic mouse brain, but differentiate in restricted foci. Development. 1996;122(7):2059-69 PMID 8681787.

34. Pegueroles J, Vilaplana E, Montal V, Sampedro F, Alcolea D, Carmona-iragui $M$, et al. Alzheimer's disease neuroimaging initiative. Longitudinal brain structural changes in preclinical Alzheimer disease. Alzheimers Dement. 2017;13(5):499-509. https://doi.org/10.1016/j.jalz.2016.08.010 PMID 27693189.

35. Vogt NM, Hunt JF, Adluru N, Dean DC, Johnson SC, Asthana S, et al. Cortical microstructural alterations in mild cognitive impairment and Alzheimer's disease dementia. Cereb Cortex. 2020;30(5):2948-60. https://doi.org/10.1093/ cercor/bhz286 PMID 31833550

36. Marzban EN, Eldeib AM, Yassine IA, Kadah YM, Alzheimer's Disease Neurodegenerative Initiative, for the Alzheimer's Disease Neurodegenerative Initiative. Alzheimer's disease diagnosis from diffusion tensor images using convolutional neural networks. PLoS One. 2020;15(3):e0230409. https://doi. org/10.1371/journal.pone.0230409 PMID 32208428.

37. Wen J, Samper-González J, Bottani S, Routier A, Burgos N, Jacquemont T, et al. Reproducible evaluation of diffusion MRI features for automatic classification of patients with Alzheimer's disease. Neuroinformatics. 2020. https://doi.org/10.1007/s12021-020-09469-5 PMID 32524428.

38. Fortin JP, Parker D, Tunç B, Watanabe T, Elliott MA, Ruparel K, et al. Harmonization of multi-site diffusion tensor imaging data. Neuroimage. 2017;161:149-70. https://doi.org/10.1016/j.neuroimage.2017.08.047 PMID 28826946.

39. Jack CR Jr, Bennett DA, Blennow K, Carrillo MC, Dunn B, Haeberlein SB, et al. NIA-AA research framework: toward a biological definition of Alzheimer's disease. Alzheimers Dement. 2018;14(4):535-62. https://doi.org/10.1016/j.jalz. 2018.02.018 PMID 29653606.

\section{Publisher's Note}

Springer Nature remains neutral with regard to jurisdictional claims in published maps and institutional affiliations.

Ready to submit your research? Choose BMC and benefit from:
- fast, convenient online submission
- thorough peer review by experienced researchers in your field
- rapid publication on acceptance
- support for research data, including large and complex data types
- gold Open Access which fosters wider collaboration and increased citations
- maximum visibility for your research: over 100M website views per year
At BMC, research is always in progress.
Learn more biomedcentral.com/submissions

